\title{
Effect of melittin on water diffusion and membrane structure in DMPC lipid bilayers
}

Buck, Z. N.; Torres, J.; Miskowiec, A.; Mamontov, E.; Kaiser, H.; Hansen, F. Y.; Taub, H.; Tyagi, M.; Collins, L.; Herwig, K. W.

\section{Published in:}

Epl

Link to article, DOI:

10.1209/0295-5075/123/18002

Publication date:

2018

Document Version

Peer reviewed version

Link back to DTU Orbit

Citation (APA):

Buck, Z. N., Torres, J., Miskowiec, A., Mamontov, E., Kaiser, H., Hansen, F. Y., Taub, H., Tyagi, M., Collins, L., \& Herwig, K. W. (2018). Effect of melittin on water diffusion and membrane structure in DMPC lipid bilayers. Epl, 123, [18002]. https://doi.org/10.1209/0295-5075/123/18002

\section{General rights}

Copyright and moral rights for the publications made accessible in the public portal are retained by the authors and/or other copyright owners and it is a condition of accessing publications that users recognise and abide by the legal requirements associated with these rights.

- Users may download and print one copy of any publication from the public portal for the purpose of private study or research.

- You may not further distribute the material or use it for any profit-making activity or commercial gain

- You may freely distribute the URL identifying the publication in the public portal 


\section{Effect of melittin on water diffusion and membrane structure in DMPC lipid bilayers}

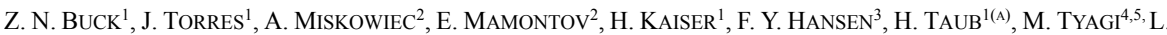
COLLINS $^{2}$, K. W. HERWIG ${ }^{2}$

1 Department of Physics and Astronomy and Research Reactor, University of Missouri, Columbia, Missouri 65211, USA

2 Center for Nanophase Materials Sciences, Oak Ridge National Laboratory, Oak Ridge, TN 37830, USA

${ }^{3}$ Department of Chemistry, Technical University of Denmark, IK 207 DTU, DK-2800 Lyngby, Denmark

${ }^{4}$ Center for Neutron Research, National Institute of Standards and Technology, Gaithersburg, MD 20899-6102I, USA

${ }^{5}$ Department of Materials Science and Engineering, University of Maryland, College Park, MD 2074, USA

received and accepted dates provided by the publisher other relevant dates provided by the publisher

PACS $87.16 . \mathrm{D}--$ Membranes, bilayers, and vesicles

PACS $78.70 . \mathrm{Nx}$ - Neutron inelastic scattering

PACS 87.14.ep-Membrane proteins

Abstract - Quasielastic neutron scattering (QENS) is well suited for studying the dynamics of water in proximity to supported membranes whose structure can also be characterized by atomic force microscopy (AFM). Here we use QENS to investigate the effect of an adsorbed peptide (melittin) on water diffusion near a single-supported zwitterionic membrane (DMPC). Measurements of the incoherent elastic neutron intensity as a function of temperature provide evidence of bulk-like water freezing onto the melittin, which AFM images indicate coalesces into peptide-lipid domains as the peptide concentration increases. Analysis of the QENS spectra indicates that, at sufficiently high melittin concentrations, a water component diffusing more slowly than bulk-like water first freezes onto the bound melittin. (794 characters with spaces; EPL $\max =800$ )

Introduction. - Membrane proteins represent a significant frontier in structural biology - they are ubiquitous in nature and perform a variety of tasks that help govern cellular activity. Their structure, insertion mechanisms, and function largely depend on the interactions between peptide-lipid domains and the hydrating water. Therefore, the dynamics of the membrane-associated water and its interaction with embedded proteins remain some of the most fundamental issues in biological physics today.

Single-supported lipid bilayer (SSLB) membranes provide model systems of biological interest for experimental investigation by a variety of techniques, including atomic force microscopy (AFM) [1] and neutron scattering [2-4]. Our recent studies have shown that quasielastic neutron scattering (QENS) is well suited for studying water dynamics associated with SSLBs and have recently used QENS to compare water diffusion in the proximity to bare membranes comprised of zwitterionic and anionic lipids [4]. We have since enhanced the complexity and biological relevance of such systems by incorporating a protein into a single-supported membrane. Here we use QENS to investigate the effect on the diffusion of water in proximity to a model peptide (melittin) bound to a singlesupported zwitterionic membrane (DMPC).
QENS has long been used to study water dynamics in the hydration layer surrounding proteins in solution [5]; however, to our knowledge, there have been no previous QENS investigations of the water dynamics near a protein bound to a bilayer phospholipid membrane. On the other hand, nuclear magnetic resonance (NMR), employing Overhauser dynamic nuclear polarization (ODNP)-enhanced NMR relaxometry, has recently been developed to probe the local translational diffusion of hydration water around specific sites of biological samples under physiological conditions [6]. This technique was used to investigate the water diffusivity within $5-10 \AA$ of spin labels tethered to lipid bilayers forming small unilamellar vesicles with proteins bound to them [6,7]. After presenting our results, we discuss how QENS measurements on singlesupported membranes at various temperatures combined with AFM imaging can complement ODNP-enhanced NMR for investigating water dynamics near membrane-bound proteins.

Melittin, the principal toxic component in bee venom, is a well-studied antimicrobial peptide (AMP) known to insert readily in DMPC membranes and has served as a prototype for understanding other pore-forming peptides [8]. Early measurements by Terwilliger et al. showed by $\mathrm{x}$-ray diffraction [9] that each melittin chain consisting of 26 amino acids adopts a mostly $\alpha$ helical conformation. Its shape can be described as 
a bent rod with two linear $\alpha$ helices joined near the middle at a 'hinge', making an angle of $\sim 120^{\circ}$. This angle can vary depending on the environment and has been measured to be as large as $\sim 160^{\circ}$ when melittin is bound to DMPC bilayers [10] The conformation and amphiphilic properties of melittin and similar AMPs result in several possible mechanisms for insertion $[11,12]$.

Terwilliger et al. proposed the so-called "wedge effect," describing how melittin adsorbs at low concentrations with its principal helical axis parallel to the bilayer surface [9]. Hydrophobic residues oriented mainly toward the inside of the helical bend penetrate the lipid head group region, while hydrophilic residues on the outside of the bend tend to orient away from the membrane center. As melittin concentration increases and aggregates form [13], the area of the outer leaflet of the membrane increases relative to that of the inner leaflet creating tension in the membrane, which can be released by the formation of transmembrane pores lined by the amphiphilic melittin peptide $[\mathbf{9 , 1 4}]$. Based on their analysis of $\mathrm{x}$-ray diffraction measurements on peptide-lipid multilayers, Lee $e$ al. [14] have argued that the wedge model of Terwilliger et al. [9] applies to transient pores in vesicles through which melittin redistributes to both sides of the membrane prior to forming stable pores. It is such stable pores induced by AMPs which have been studied in measurements of liposome leakage kinetics [15].

The question that we address here is how the structure of the DMPC membrane and the diffusion of its hydration water are affected by the binding of melittin. Our AFM measurements provide evidence of melittin aggregation on the membrane surface with increasing melittin concentration. To establish the interaction of the hydration water with the adsorbed melittin, we demonstrate that the presence of melittin strongly affects the water's freezing behavior compared to that in proximity to a bare DMPC membrane. Analysis of the QENS spectra provides evidence that, at sufficiently high melittin concentrations, water component diffusing more slowly than bulk-like water first freezes onto the bound melittin.

Methods. - Our sample fabrication began by using a vesicle fusion method identical to that described previously $[4,16,17]$ to deposit uniform, single, bare DMPC membranes on singlecrystal silicon wafers ( $5 \mathrm{~cm}$ diameter and $0.3 \mathrm{~mm}$ thick) coated with their native oxide $\left(\mathrm{SiO}_{2}\right)$. These samples were rinsed with deionized water to remove lipid material above the complete DMPC bilayer adjacent to the $\mathrm{SiO}_{2}$ surface. Melittin $\left(\mathrm{C}_{131} \mathrm{H}_{229} \mathrm{~N}_{39} \mathrm{O}_{31}\right)$ powder purchased from Sigma Aldrich ${ }^{1}$ and Gen Script ${ }^{1}$ (purity $>96 \%$ ) was used for the AFM samples and melittin from Sigma Aldrich (purity $>85 \%$ ) was used for the QENS samples. The melittin was dissolved in an aqueous solution to form $600 \mu \mathrm{M}$ aliquots, which were stored at $253 \mathrm{~K}$ until further use. Upon thawing, these aliquots were diluted to obtain solutions with peptide concentrations ranging from 0.1 $\mu \mathrm{M}$ to $1.0 \mu \mathrm{M}$ in a buffer containing $10 \mathrm{mM}$ HEPES and 150 $\mathrm{mM} \mathrm{NaCl} \mathrm{pH} \mathrm{7.2.} \mathrm{These} \mathrm{salt} \mathrm{concentrations} \mathrm{were} \mathrm{found} \mathrm{to} \mathrm{be}$ optimal in facilitating the deposition of homogenous melittin- treated single-supported bilayers [18]. The previously prepared single-supported DMPC bilayers were submerged in the peptide solution and incubated at $328 \mathrm{~K}$ for 4 hours. The wafers were periodically flipped and the solutions gently stirred during deposition in order to ensure all the available membrane surface area was exposed to the peptide solution. We note that similarly prepared melittintreated bilayers have been characterized in-situ using a quartz-crystal microbalance where the observed time scale over which such peptide-membrane interactions occurred was measured to be on order of several hours for the same temperature that we have used [19]. After their exposure to the peptides, the wafers containing melittin-treated DMPC bilayers were carefully removed from the solution, rinsed with deionized water, and then placed in aluminum drying trays.

To control the hydration level of our samples fabricated for neutron scattering experiments, we annealed the melittintreated DMPC membranes in air at $328 \mathrm{~K}$ in order to evaporate excess water. A stack of 100 wafers was then sealed in an aluminum sample can under a helium atmosphere with a known amount of water $\left(120 \mu \mathrm{H} \mathrm{H}_{2} \mathrm{O}\right)$ [4] to ensure full hydration of the membranes. Before sealing the samples, we used atomic force microscopy (AFM) to confirm complete membrane coverage and sample homogeneity.

AFM. - Topographical images from melittin-treated DMPC bilayers fabricated for neutron scattering measurements were collected under ambient conditions using a Digital Instruments Nanoscope IIIa AFM at the University of Missouri. Imaging of the melittin-treated membranes were performed before and after they had been subjected to an anneal at $328 \mathrm{~K}$. To visualize structural changes to the membrane induced by melittin during deposition, we also performed in situ imaging of similarly prepared bilayers supported on mica substrates using a Cypher ES Environmental AFM at the Center for Nanophase Material Sciences at Oak Ridge National Laboratory. In both cases, AFM images were recorded under tapping mode using silicon nitride tips (radius $<10 \mathrm{~nm}$ ) possessing spring constants and resonant frequencies in the range of $0.1-0.3 \mathrm{~N} /$ $\mathrm{m}$ and $20-40 \mathrm{kHz}$, respectively.

In situ AFM measurements were first conducted on a bare DMPC bilayer in order to confirm sample quality. Images of the bare membrane prior to its exposure to melittin show a featureless surface with an average roughness slightly greater than that of the mica substrate $(<0.1 \mathrm{~nm})$. After imaginb bare DMPC, the buffer was carefully removed and replaced with a new solution containing a melittin concentration of $1.0 \mu \mathrm{M}$. Figure 1 shows two in situ AFM images collected at $303 \mathrm{~K}$ from (a) a bare DMPC bilayer supported on mica under buffer

${ }^{1}$ Certain commercial equipment, instruments, or materials (or suppliers) are identified in this paper to foster understanding. Such identification does not imply recommendation or endorsement by the National Institute of Standards and Technology, nor does it imply that the materials or equipment identified are necessarily the best available for this purpose. 
solution and (b) the same sample 60 minutes after exposure to melittin.

After this exposure, dimple-like features can be seen on the DMPC surface (fig. 1(b)), which occupy roughly $6-10 \%$ of the total scan area. Line trace analysis over the dimples reveals diameters in the range of $60-80 \mathrm{~nm}$ and depths of roughly $1 \mathrm{~nm}$. Upon further exposure, the dimple-like features remained stable for the rest of the measurement $(\sim 80$ $\min$ ) before the buffer solution evaporated.

The lower two panels in Fig. 1 show the effect of annealing on DMPC membranes treated with a solution containing 1.0 $\mu \mathrm{M}$ melittin. Unlike those in the upper panels, these AFM images were taken in the absence of buffer solution and at a slightly lower temperature of $295 \mathrm{~K}$. Prior to annealing [Fig. $1(\mathrm{c})$ ], we again observe dimple-like features on the membrane surface as in Fig. 1(b), although the longer exposure time of 4 hours and drier imaging conditions yield larger diameters $(\lesssim$ $200 \mathrm{~nm}$ vs. $\sim 70 \mathrm{~nm}$ ). Despite the drier state of the membrane, the diameters increase with annealing up to $500 \mathrm{~nm}$ [Fig. 1(d)], but the dimples maintain about the same depth of $\sim 1 \mathrm{~nm}$ as those observed initially and in the in-situ case.

The annealing temperatures for our melittin-treated samples were determined previously from AFM temperature dependent studies of the DMPC bilayer thickness in air, showing a fluid phase transition at $328 \mathrm{~K}$ [17]. Although during its anneal the membrane is in a dehydrated state, the lipid motion is more rapid at these higher temperatures. This increased mobility is believed to facilitate the aggregation of surface-bound melittin, a hypothesis supported by measurements of the incoherent elastic neutron scattered intensity as a function of time [17]. The elastic intensity of SSLB DMPC membranes treated with melittin concentrations of $0.5 \mu \mathrm{M}$ were found to increase by as much as $5 \%$ during annealing at $328 \mathrm{~K}$ over a period of several hours. Annealing times exceeding 8 hours resulted in saturation of the elastic neutron intensity, indicating no further impedance of the $\mathrm{H}$ atom motion in the samples. This behavior is consistent with the diffusion and subsequent anchoring of melittin into domains, such as those observed in Fig. 1(d).

Previous studies suggest that, prior to forming transmembrane pores, melittin and other AMPs can induce thinning of the membrane [20-22]. Mecke et al. [1] performed AFM measurements on supported DMPC bilayers treated with an AMP similar to melittin at concentrations of 1-10 $\mu \mathrm{M}$. They also observed formation of distinct domains of a peptide-lipid phase of a well-defined thickness less than that of the surrounding bilayer and which was independent of the total amount of peptide bound to the membrane [1]. These authors found the average height difference of these domains to be $1.1 \pm 0.2 \mathrm{~nm}$ comparable to the $\sim 1 \mathrm{~nm}$ depth of the dimple-like features in Fig. 1. In addition, Rakowska et al., [23] have reported AFM images collected in air from a supported PC bilayer treated with a synthetic AMP that reveal circular impressions in membrane as large as $10 \mu \mathrm{m}$ in diameter and depths of less than $<1 \mathrm{~nm}$ as a result of its exposure to a $10 \mu \mathrm{M}$ peptide solution.
We analyzed AFM images of samples similar to those used for our QENS measurements following the procedure that Mecke et al. used to infer a peptide-to-lipid ratio $P / L[\mathbf{1}]$. To estimate $P / L$ for our samples, each pixel of the AFM images in Fig.1 was binned according to whether it belonged to a dimple or not. Knowing the total area occupied by the dimples, we then calculated an approximate $P / L$ under the following assumptions: 1) the melittin peptides are bound to the DMPC membrane with their helical axis parallel to the bilayer plane $[\mathbf{9 , 1 4}] ; 2)$ the peptide and the lipid occupy a cross-sectional area of $4 \mathrm{~nm}^{2}$ [9] and $0.6 \mathrm{~nm}^{2}$ [1], respectively; 3) within a dimple, the peptides completely displace lipids from the upper leaflet as in Fig. 7(c) of Ref. 1; and 4) there are a negligible number of peptides outside the dimples. The constancy observed for the dimple depth supports assumption 3 ). If a varying number of lipids remained in the upper leaflet within the dimple areas, we might expect a distribution of dimple depths. With regard to assumption 4), we note that the presence of peptides between dimples would increase our estimate of $P / L$; however, they are likely to exist as isolated monomers or small clusters too small to participate in pore formation.
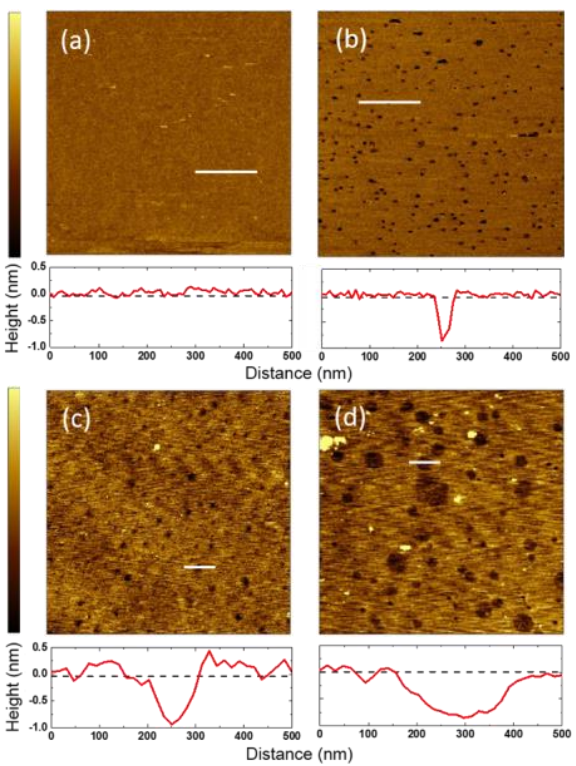

Figure 1: AFM images and line scans of $1.0 \mu \mathrm{M}$ melittintreated DMPC supported bilayers. The upper panel displays in situ images of a DMPC membrane (a) collected under buffer at $303 \mathrm{~K}$ before the introduction of melittins and (b) after 60 minutes of exposure to melittin. AFM images in the bottom panel were collected in air at $295 \mathrm{~K}$ (c) before and (d) after annealing at $328 \mathrm{~K}$. Line scans were performed over the 0.5 $\mu \mathrm{M}$ white scale bar shown in the images and reveal depths of roughly $1 \mathrm{~nm}$ for all dimple-like features. Upon annealing as shown in (d), the dimple diameters increase to as large as 500 $\mathrm{nm}$ without destroying the membrane. All images were collected in tapping mode and have a z-scale of $1 \mathrm{~nm}$. 
Fig. 1: AFM images and line scans of $1.0 \mu \mathrm{M}$ melittin-treated DMPC supported bilayers. The upper panel displays in situ images of a DMPC membrane deposited on a mica substrate collected under buffer at $303 \mathrm{~K}$ (a) 0 minutes and (b) 60 minutes after an exposure to melittin. AFM images in the bottom panel were collected in air at $295 \mathrm{~K}$ from samples deposited on silicon wafers (c) before and (d) after an anneal a $328 \mathrm{~K}$. Line scans were performed over the $0.5 \mu \mathrm{m}$ white scale bar shown in the images and reveal depths of roughly $1 \mathrm{~nm}$ for all dimple-like features. Upon annealing (d) the dimple diameters increase to as large as $500 \mathrm{~nm}$ without causing membrane destruction. All images were collected in tapping mode and have a $z$-scale of $1 \mathrm{~nm}$.

Under these assumptions, we estimate a maximum peptideto-lipid ratio $(P / L)$ of $\sim 1 / 120$ for the $1.0 \mu \mathrm{M}$ melittin-treated sample in Fig. 1(d) and 1/300 for a similarly prepared $0.5 \mu \mathrm{M}$ sample. Despite the uncertainty in these estimates, we conclude that our SSLB QENS sample made with a $0.5 \mu \mathrm{M}$ melittin concentration is substantially below the critical value of $P / L^{*}=$ $1 / 45$ determined for pore formation in DMPC multilayer membranes exposed to melittin [14]. Therefore, in our analysis of the QENS spectra, we consider the possibility of wate confined to pores for a $0.5 \mu \mathrm{M}$ melittin-treated sample to be unlikely; however, we cannot completely exclude their presence. Our value of $P / L$ for this sample allows us to estimate the number of $\mathrm{H}$ atoms in the peptide $(\sim 0.03 \%)$, the lipids $(\sim 3 \%)$, and the membrane-associated water $(97 \%)$.

DMPC bilayers treated with melittin concentrations greater than $1.0 \mu \mathrm{M}$ were also investigated by AFM. However, their surfaces were found to be highly disordered with insufficient homogeneity for neutron scattering measurements.

Elastic Neutron Scans. - The large incoherent crosssection of hydrogen and the preponderance of $\mathrm{H}$ atoms in the water contained in our samples results in water molecules providing the dominant contribution to our QENS spectra Before performing time-consuming QENS measurements, it is useful to measure the temperature dependence of the intensity of neutrons scattered elastically from a sample. These elastic scans aid in the identification of temperature ranges at which to collect QENS spectra for investigating the water dynamics. We conducted these elastic scans on the High Flux Back Scattering Spectrometer (HFBS) at NIST Center for Neutron Research (NCNR) in Gaithersburg, MD [24]. Its energy resolution of $\sim 1$ $\mu \mathrm{eV}$ allows only molecular motions occurring on a time scale longer than $\sim 4 \mathrm{~ns}$ to contribute to the elastic intensity. Thus, an increase in the elastic intensity is proportional to the amount of frozen water in a sample.

Figure 2 shows the temperature dependence of elasticallyscattered neutrons from water near melittin-treated DMPC membranes upon cooling. Intensities were summed over all 16 detectors of the HFBS, spanning a wave vector transfer range $0.25 \AA^{-1}<Q<1.75 \AA^{-1}$, and normalized to unity at temperature of $275 \mathrm{~K}$. The elastic scan from the sample made from the solution with the lowest melittin concentration, 0.1 $\mu \mathrm{M}$, closely resembles that of the bare DMPC membrane (black squares in Fig. 2) except that the bulk-water freezing transition at $265 \mathrm{~K}$ is now slightly broadened. We believe that this broadening results from a dilute dispersion of melittin monomers on the membrane surface. The broadened step in the elastic scan is similar to that which is observed for the freezing of bulk-like water near a bare DMPG membrane, which is more hydrophilic than DMPC [4]. Note the lower hydration of the $0.1 \mu \mathrm{M}$ sample compared to the bare DMPC sample as indicated by the lower plateau in intensity at low temperature.

As the melittin concentration used to treat the DMPC membranes increases to $0.5 \mu \mathrm{M}$, a sharp freezing transition of the membrane-associated water appears at $270 \mathrm{~K}$ (green circles in Fig. 2); and, concomitantly, there is decrease in the height of the freezing step of bulk-like water observed at $265 \mathrm{~K}$ for the intensity at $270 \mathrm{~K}$ increases nearly in proportion to the melittin

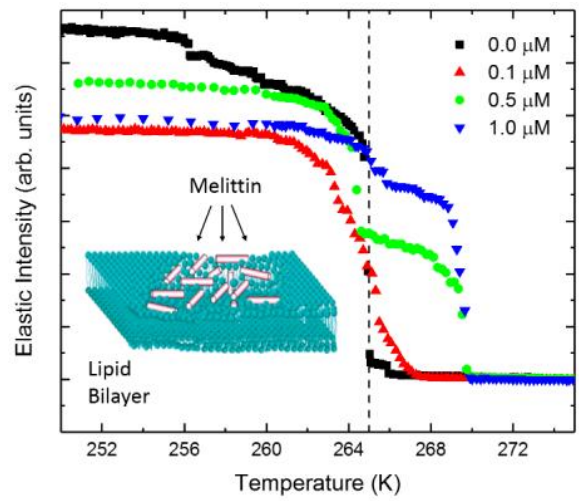

Fig. 2: Elastic neutron scans measured on the HFBS upon cooling of melittin-treated DMPC bilayers. Scans of four samples made from solutions of different melittin concentrations are shown. Intensities have been summed over all $Q$ and normalized to unity at $275 \mathrm{~K}$. Samples were continuously cooled at a rate of $0.04 \mathrm{~K} / \mathrm{min}$ over the temperature range $260 \mathrm{~K}<\mathrm{T}<275 \mathrm{~K}$ with data being collected every 5 minutes. Data on the bare DMPC membrane $(0.0 \mu \mathrm{M})$ are taken from Ref. 2. The vertical dashed line at $265 \mathrm{~K}$ indicates the temperature at which bulk-like water freezes in the bare-membrane sample. The inset depicts the structure of a DMPC membrane treated with high melittin concentrations as adapted from Ref. 11.

concentration. Upon further cooling, the bulk-like wate freezing transition identified in the bare-membrane sample is recovered around $265 \mathrm{~K}$, but with a smaller step upward in intensity. We propose that the newly observed freezing transition at $270 \mathrm{~K}$ results from water freezing onto melittin domains of the type observed in the AFM images of Fig. 1 and as depicted in the inset of Fig. 2. 
In the case of the bare DMPC membrane, all water melted by $272 \mathrm{~K}$, i.e., below the melting point of bulk ice at $273 \mathrm{~K}$. However, upon heating, the elastic intensity for all three melittin-treated DMPC membranes displays a single sharp melting transition slightly above the bulk-ice melting point that depends on melittin concentration during incubation: $273.3 \mathrm{~K}$ $(0.1 \mu \mathrm{M})$ and $273.7 \mathrm{~K}(0.5$ and $1.0 \mu \mathrm{M})$. This melting behavior provides further evidence that water is interacting strongly with the membrane-bound melittin.

Quasielastic Spectra. - Full quasielastic spectra were collected from melittin-treated DMPC bilayers on the Backscattering Silicon Spectrometer (BASIS) located at the Spallation Neutron Source (SNS) at Oak Ridge National Lab (ORNL) [23]. We chose the $0.5-\mu \mathrm{M}$ melittin sample for further investigation based on the observation in the elastic scans of two distinct freezing transitions of the membrane-associated water at $270 \mathrm{~K}$ and $265 \mathrm{~K}$ that had comparable changes in elastic intensity (green triangles in Fig. 2) and were reproducible over multiple thermal cycles (data not shown) [17]

On cooling of the melittin-treated sample, we obtained QENS spectra with counting times of 1 hour at a temperature interval of $0.5 \mathrm{~K}$ over a temperature range that included the freezing transitions. The spectra were fitted using the DAVE software [26] by folding the instrumental resolution function with a scattering law composed of three terms: a delta function corresponding to the elastic scattering and two Lorentzian terms representing the quasielastic scattering.

The large dynamic range of BASIS $( \pm 120 \mu \mathrm{eV})$ and its $Q$ range $\left(0.3 \AA^{-1}<Q<1.9 \AA^{-1}\right)$ comparable to that of the HFBS allows us to resolve two distinct diffusive processes occurring on different time scales: a "fast" motion described by a broad Lorentzian and a "slow" motion represented by a narrow Lorentzian in addition to the elastic component. The temperature dependence of the three spectral components is plotted in Fig. 3. In panel (a), we see that the elastic component is in qualitative agreement with that measured with a different sample on the HFBS (cf. Fig. 2, green circles). On cooling, there is again an abrupt upward step in the elastic intensity at $269 \mathrm{~K}$, which we have attributed to bulk-like water freezing onto the peptide. It is followed by a wider step near $267 \mathrm{~K}$, which is somewhat weaker and broader than the one at $265 \mathrm{~K}$ in the HFBS elastic scan. We attribute these differences to a greater heterogeneity in the BASIS sample, its somewhat lower purity $(85 \%)$, and possibly differences in thermometry and method of temperature control. The elastic scans on the HFBS were collected continuously with their intensities averaged over 5 minutes with a cooling rate of $0.04 \mathrm{~K} / \mathrm{min}$ whereas the BASIS data were obtained at a fixed temperature for 1 hour.

Figure 3(b) shows that a step-like decrease in the intensity of the broad Lorentzian occurs at the same temperature as the step-like increase in the elastic component (Fig. 3(a)) that was attributed to the freezing of bulk-like water onto the peptide. At lower temperature, there is a plateau in intensity followed by a more gradual decrease in the broad Lorentzian intensity, matching the wider step upward in the elastic intensity near 267 $\mathrm{K}$. Based on this correspondence between the intensities of the elastic and broad-Lorentzian components in the QENS spectra, we identify the broad Lorentzian as representing the diffusion of bulk-like water. Its temperature dependence below $269 \mathrm{~K}$ may result from the freezing of bulk-like water over regions of the DMPC membrane unoccupied by melittin. We discuss below the interpretation of the narrow Lorentzian component in Fig. 3(c).

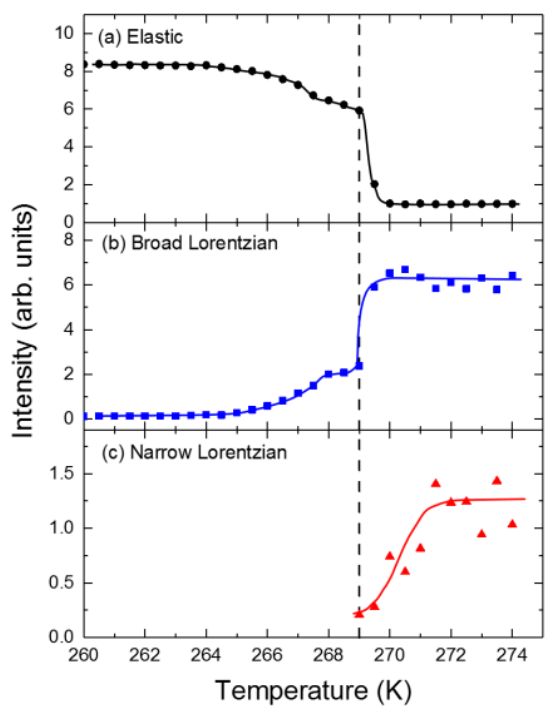

Fig. 3: Temperature-dependence of the intensity of the three components in the QENS spectra from $0.5 \mu \mathrm{M}$ melittin-treated DMPC measured on BASIS upon cooling. (a) The elastic intensity has been summed over all $Q$ and normalized to unity at $275 \mathrm{~K}$; (b) integrated intensity of the broad-Lorentzian component representing fast motion; and (c) integrated intensity of the narrow-Lorentzian component representing slow motion. Both quasielastic terms have been summed over the $O$ values used to obtain the diffusion coefficients, $0.5 \AA^{-1}$, $0.7 \AA^{-1}$, and $0.9 \AA^{-1}$ and normalized to the elastic intensity at $275 \mathrm{~K}$. The vertical black dashed line at $269 \mathrm{~K}$ represents the temperature at which the transition attributed to the freezing of bulk-like water onto the peptides completes. The solid color lines are guides to the eye.

The half-width-at-half-maximum $(I)$ of both Lorentzian components in the BASIS spectra exhibits a $Q^{2}$ dependence at low $Q$, characteristic of translational diffusion. Performing a least squares fit to Fick's Law $\left(\Gamma=D Q^{2}\right)$ at low $Q$, we obtained a diffusion coefficient $D$ for both the broad- and the narrowLorentzian components in the spectra. The weaker intensity of 
the narrow Lorentzian (see Fig. 3) made fitting the QENS spectra with two components difficult. Therefore, in order to resolve the narrow component and infer a diffusion coefficient from it, we began our fitting procedure by fixing the width of the broad Lorentzian at values corresponding to those of bulk supercooled water at temperatures above $269 \mathrm{~K}$ [27, 28]. This constraint allowed a stable two-Lorentzian fit to the spectra initially, and subsequently it could be relaxed. We were unable to fit spectra collected below $269 \mathrm{~K}$ reliably using two Lorentzians.

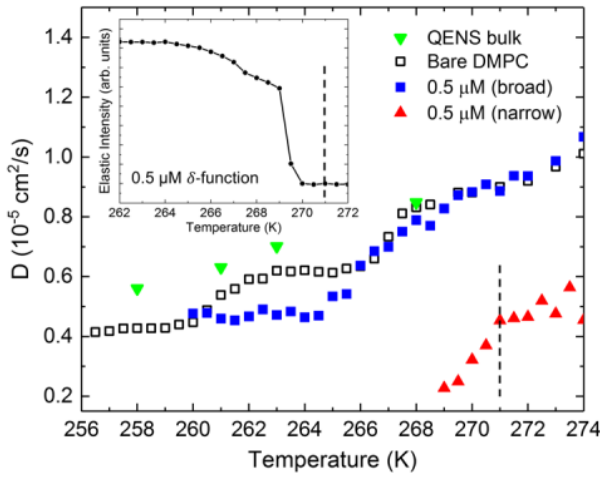

Fig. 4: Temperature dependence on cooling of the two diffusion coefficients inferred from analysis of the QENS spectra from DMPC membranes treated with $0.5 \mu \mathrm{M}$ melittin: broad Lorentzian (blue squares) and narrow Lorentzian (red triangles). For comparison, the values of $D$ from bare DMPC membranes (open black squares) from Ref. 3 and bulksupercooled water (green triangles) from Ref. 28 are also shown. The inset contains the elastic intensity of the melittintreated sample obtained on BASIS (from Fig. 3). The dashed black vertical line at $271 \mathrm{~K}$ indicates the temperature at which the diffusion coefficient associated with the narrow componen begins to decrease.

Figure 4 contains the diffusion coefficient as a function of emperature determined for bare DMPC (from Ref. 3) and for the two Lorentzian components in the spectra of the melittintreated DMPC. At temperatures above $268 \mathrm{~K}$, the diffusion coefficient obtained from the broad-Lorentzian component in the spectra of the melittin-treated sample (blue square points) closely tracks that identified with bulk-like water in the bare DMPC sample [3] as well as that of bulk supercooled water [27, 28]. This behavior is consistent with the identification of the broad-Lorentzian component with bulk-like water made from the analysis of its intensity in Fig. 3. However, below $268 \mathrm{~K}, D$ begins to deviate from the bulk behavior, decreasing steadily before leveling off at $264 \mathrm{~K}$, the same temperature at which the elastic intensity plateaus (see inset to Fig. 4). We have previously identified the plateau in $D$ of the bare DMPC membrane in the temperature range $262 \mathrm{~K}<T<265 \mathrm{~K}$ with a water type termed "confined 1 ," which remains mobile below the freezing transition of bulk-like water and which may be located in the region between bulk ice and the lipid head groups [4]. The discrepancy with the diffusion coefficient inferred from the broad Lorentzian of the melittin-treated sample in this temperature range suggests that the confined 1 water also freezes onto the peptide.

The diffusion coefficient inferred from the narrowLorentzian component (red triangles in Fig. 4) has a magnitude roughly half that of the bulk-like component at $273 \mathrm{~K}$ and displays a qualitatively different temperature dependence, which tracks that of the narrow-Lorentzian intensity in Fig. 3 (c). Above $271 \mathrm{~K}, D$ is constant to within the measured uncertainty, but at lower temperatures decreases to a value 0.22 $\times 10-5 \mathrm{~cm}^{2} / \mathrm{s}$ at $\sim 269 \mathrm{~K}$ where the intensity of the narrow component vanishes. We suggest that this more slowly diffusing water population is located closer to the melittin aggregates and interacts more strongly with them than the bulklike water.

Conclusion. - We have investigated the diffusion of water in proximity to a DMPC membrane on which the peptide melittin has been deposited. Our AFM measurements indicate that the melittin coverage is sufficient for it to coalesce into domains whose thickness is $\sim 1 \mathrm{~nm}$ less than that of the surrounding membrane. However, the melittin coverage is estimated to be below the threshold $P / L$ ratio necessary for pore formation. At this concentration, melittin strongly affects the freezing behavior of the membrane-associated water to the extent that water, sufficiently far from the membrane to diffuse at a rate close to that of bulk water, freezes onto the peptide. In addition, we find evidence of a second water component, which has a translational diffusion coefficient about half of that of the bulklike water and which begins to freeze onto the peptide at a temperature a few degrees above the bulk-like water. We speculate that larger proteins may exhibit more than the two freezing transitions that we have found for water onto melittin and thereby reveal the relative strength of different binding sites for water. Also, a more slowly diffusing water component in proximity to the inserted peptide may be a feature common to other non-pore-forming antimicrobial peptides.

Our results suggest a role for QENS measurements on SSLBs in complementing the ODNP-enhanced NMR technique for investigation of water dynamics near membranebound proteins. In addition to the need to determine the effect of the spin-label on the system of interest, the NMR technique is limited to room-temperature measurements. By performing QENS measurements at lower temperatures, it is possible to freeze out different types of motion such as that of bulk water. Also, in some cases, it may be desirable to probe the water dynamics beyond 5-10 $\AA$ from the spin label. Although a QENS measurement involves an average over a macroscopic sample, the wave vector and energy dependence of the QENS spectra allow one to separate molecular motions from distinct water populations characterized by their respective length and time 
scales. Therefore, by combining AFM with QENS measurements on single-supported membrane samples of sufficient homogeneity, one is able to probe both the structure of the bound peptides and the dynamics of the membraneassociated water on the nanoscale.

\section{$* * *$}

This work was supported by the U.S. National Science Foundation under Grant No. DGE-1069091 and utilized facilities supported in part by the NSF under agreement No. DMR-1508249. Z. N. B. was partially supported by the U.S Department of Energy Office of Science Graduate Student Research (SCGSR) program under contract number DESC0014664. A portion of this research at Oak Ridge National Laboratory's Spallation Neutron Source was sponsored by the Scientific User Facilities Division, Office of Basic Energy Sciences, U.S. Department of Energy. We thank Gavin King, Ioan Kosztin, and Dan Neumann for helpful discussions.

\section{REFERENCES}

[1] Mecke A., Lee D. K., Ramamoorthy A., Orr B. G. and Holl M. M., Biophys. J., 89 (2005) 4046.

[2] Bai M., Miskowiec A., Hansen F. Y., Taub H., Jenkins T., Tyagi M., Diallo S. O., Mamontov E. Herwig K. W., and Wang S.-K., EPL, 98, (2012) 48006.

[3] Miskowiec A., Buck Z. N., Brown M. C., Kaiser H. Hansen F. Y., King G.M., Taub H., Jiji R., Cooley J. W., Tyagi M., Diallo S. O., Mamontov E., and Herwig K. W., EPL, 107 (2014) 28008

[4] MisKOWIEC A. BUCK Z. N., KAISER H., HANSEN F. Y., TAub H., Tyagi M., Diallo S. O., Mamontov E., AND Herwig K. W., J. Chem. Phys., 146 (2017) 125102

[5] Schirò G., Fichou Y., Gallat F.-X., WoOd K., GABEl F., MOUlin M., HÄRTLEIN M., HEYdEN M., Colletier J.-P., Orecchini A., PACIARONi A. WutTKE J., ToBias D. J., AND WeIK M., Nature Comm., 6 (2015) 6490.

[6] Cheng C.-Y., VArkey J., Ambroso, M. R., Langen R., And Han S., Proc. Natl. Acad. Sci. U.S.A., 110 (2013) 16838.

[7] Fisett O., Päslack C., Barnes R., Isas J. M., Langen R., Heyden M., Han S., and Schäfer L. V., J. Am. Chem. Soc., 138 (2016) 11526.

[8] Raghuraman H. and Chattopadhyay A., Bioscience Report, 27 (2007) 189.

[9] Terwilliger T. C., Weissman L., and Eisenberg D. Biophys. J., 37 (1982) 354

[10] Naito A., Nagao T., Norisada K., Mizuno T., Tuzi S., Saito H., BioPhys. J., 78 (2000) 2405.

[11] Wimley W., ACS Chemical Biology, 5 (2010) 909.

[12] Brogden K.A., Nature review Microbiology., 3(3) (2005) 243.

[13] Klocek G., Schulthess T., Shai Y., Seelig J., Bio Chem., 48 (2009) 2586.
[14] Lee M.-T., Sun T.-L., Hung W.-C., Huang H. W., Proc. Natl. Acad. Sci. U.S.A., 110 (2013) 14245.

[15] Last N. B., Miranker A.D., Proc. Natl. Acad. Sci. U.S.A., 110 (2013) 6382

[16] Miskowiec, Ph.D. Thesis, University of Missouri, 2014 (unpublished).

[17] Buck Z. N., Ph.D. Thesis, University of Missouri, (2018).

[18] Sugihara, K., Delai, M., Szendro, I., GuillaumeGentil, O., Vörös, J., and Zambelli, T., Sensors and Actuators B: Chemical, 161 (2012) 601.

[19] Lu N., Yang K., Yuan B., and Ma Y., J. Phys. Chem. B., 116(31) (2012) 9432-8.

[20] Chen F. Y., Lee M. T., and Huang H. W., Biophys. J., 84 (2003) 3754

[21] Huang H. W., Chen F.Y., and Lee M. T., Phys. Rev. Lett., 92 (2004) 198304.

[22] Ludtke S., He K., and Huang H., Bio. Chem., 34 (1995) 16767.

[23] Rakowska P. D., Jiang H., Ray S., Pyne A., Lamarre B., Carr M., Judge P J., Ravi J., Gerling U. I. M., Koksch B., Martyna G. J., Hoogenboom B. W., Watts A., Crain J., Grovenor C. R. M., and Ryadnov M. G., Proc. Natl. Acad. Sci. U.S.A., 110 (2013) 8921.

[24] Meyer A., Dimeo R.M., Gehring P.M., Neumann D.A., Rev. Sci. Instrum., 74 (2003) 2759

[25] Mamontov E. and Herwig K. W., Rev. Sci. Instrum., 82 (2011) 085109

[26] Azuah R.T, Kneller L.R., Qiu Y., Tregenna-Piggott P.L. W., Brown C.M., Copley J.R.D., and Dimeo R.M., J. Res. Natl. Inst. Stan. Technol., 114 (2009) 341.

[27] Teixeira J., Bellissent-Funel M.-C., Chen S. H., and Dianoux A. J., Phys. Rev. A, 31 (1985) 1913.

[28] Price W. S., Ide H., and Arata Y., J. Phys. Chem. A, 103 (1999) 448. 